\title{
Dynamic friction energy dissipation and enhanced contrast in high frequency bimodal atomic force microscopy
}

\author{
Xinfeng TAN, Dan GUO*, Jianbin LUO* \\ State Key Laboratory of Tribology, Tsinghua University, Beijing 100084, China \\ Received: 24 September 2020 / Revised: 20 December 2020 / Accepted: 07 January 2021 \\ (C) The author(s) 2021.
}

\begin{abstract}
Dynamic friction occurs not only between two contact objects sliding against each other, but also between two relative sliding surfaces several nanometres apart. Many emerging micro- and nano-mechanical systems that promise new applications in sensors or information technology may suffer or benefit from noncontact friction. Herein we demonstrate the distance-dependent friction energy dissipation between the tip and the heterogeneous polymers by the bimodal atomic force microscopy (AFM) method driving the second order flexural and the first order torsional vibration simultaneously. The pull-in problem caused by the attractive force is avoided, and the friction dissipation can be imaged near the surface. The friction dissipation coefficient concept is proposed and three different contact states are determined from phase and energy dissipation curves. Image contrast is enhanced in the intermediate setpoint region. The work offers an effective method for directly detecting the friction dissipation and high resolution images, which overcomes the disadvantages of existing methods such as contact mode AFM or other contact friction and wear measuring instruments.
\end{abstract}

Keywords: dynamic friction; energy dissipation; contact states; image contrast; bimodal atomic force microscopy (AFM)

\section{Introduction}

Friction is very common in the macro world, but the explanation of the origin is still scarce and controversial [1-5]. Atomic force microscopy (AFM), an innovative technique invented in 1986, has become a multifunctional and powerful apparatus for imaging nanometer resolution surface structures and friction measurement in various environments [6-13]. The first atomic-scale feature of a graphite surface was observed as the stick-slip phenomenon in the friction force microscopy (FFM), and some other two-dimensional materials were proved to be in superlubricity state and exhibit excellent lubricant properties as additives at microscale [14-16]. Friction traced to the single atom on Si(111)-(7×7) was obtained by a qPlus sensor in the frequency modulation (FM) AFM, a dynamic mode for the real noncontact friction detection [17]. Although the resolution has reached a sub-atomic level, this technique is only capable to measure either the lateral or the normal force [18]. How to measure two-directional interaction by the tip is still a challenge issue in the development of AFM technology. An optional force sensor is a microcantilever beam, which can flexibly detect the two directional interaction by theoretically exciting both the flexural and torsional vibration modes [19, 20]. Piezoelectric actuation is a commonly used method, however, it has the problem of spurious flexural resonances. To avoid the "forest of peaks", some other excitation methods, such as the photothermal and magnetic actuations have been developed [21-23]. In recent years, the bimodal technique

* Corresponding authors: Jianbin LUO, E-mail: luojb@tsinghua.edu.cn; Dan GUO, E-mail: guodan26@tsinghua.edu.cn 
[24-26] or higher modes [27, 28], multi-frequency $[29,30]$, and intermodulation method have enriched the conventional dynamic force microscopy (DFM) field, thereby greatly improving the image resolution and obtaining more sample information [31-35].

The first bimodal method excites the first two modes of the microcantilever to explore interface interaction with high precison [24]. However, sometimes the friction or lateral dissipation provides additional information in the heterogeneous materials and some laminated structures [36]. The bimodal AFM method turned out to be an effective method for the vertical and lateral detection [37, 38]. Recently, in the amplitude modulation flexural-frequency modulation torsional (AMFlex-FMTor) mode DFM, the in- and out-of-plane nanomechanical properties of two heterogeneous polymers were obtained [39]. However, in atmosphere environment, the cantilever with small amplitude and force constant does not seem to be an appropriate choice for stable feedback, mainly because the water film or the force field on the sample can attract the tip into the sample surface [40]. It can be addressed through exciting the second order flexural vibration modes as the feedback since the second order force constant is about 40 times higher than the first order, which allows the tip to vibrate stably near the sample surface and overcomes the pull-in instability. The stable vibration in the tapping mode is also a key for understanding the energy dissipation mechanism near the surface. In 2006, Socoliuc et al. experimentally controlled the friction by changing the load of nano-contact, and even ultra-low friction can be achieved [41]. Then efficient methods to control superlubricity by external vibration conditions have been developed [42]. The energy dissipation in the tapping mode seems to be deeply related to the vibration induced superlubricity due to the similar motion patterns [43].

In this work, a bimodal approach combining the second order flexural and the first order torsional eigenmodes of the cantilever is introduced to detect the contrast, the in- and out-of-plane dissipation between the tip and the heterogeneous polymers. In this way, the dynamic friction energy dissipation near the surface can be studied in depth. Besides, the friction dissipation coefficient is firstly proposed and three different contact states are determined from the energy dissipation and phase curves. When the second order flexural vibration is used as feedback to measure samples at close range, the image contrast can be greatly enhanced.

\section{Experiments}

The original experiment setup has been described in the previous study [40]. Figure 1 depicts the high order bimodal AFM schematic along with the observables, the second order flexural $\left(f_{2}=1,800.1\right.$ $\mathrm{kHz}$, ) and the first order torsional $\left(f_{\mathrm{tr}}=2,321.5 \mathrm{kHz}\right)$ vibration signal spectra. The bimodal vibration was excited by the piezoelectric actuator, in which the high order feedback was applied for the steady control [19]. The sample was the polymer blend of polystyrene $(\mathrm{PS})\left(E_{\mathrm{PS}}=2.0 \mathrm{GPa}\right)$ and low-density polyethylene $(\mathrm{LDPE})\left(E_{\mathrm{LDPE}}=0.1 \mathrm{GPa}\right)$ purchased from Bruker Corporation (HarmoniX Training Sample), which is a commonly used sample to display the bimodal AFM functions [44, 45]. The inverse optical lever sensitivities (InvOLS) were calibrated from dynamic amplitude approaching curves on the silicon surface for the second order flexural mode [46, 47]. Therefore, the flexural and lateral amplitude sensitivity were 10.52 and 3.51 $\mathrm{nm} / \mathrm{nA}$, respectively. The cantilever used here was $\mathrm{PPP}-\mathrm{NCH}$ type, and the first, second order flexural and first order torsional force constants were calibrated by Sader's method $\left(k_{1}=21.6 \mathrm{~N} / \mathrm{m}, k_{2}=\right.$ $844.1 \mathrm{~N} / \mathrm{m}, k_{\mathrm{tr}}=1,403.8 \mathrm{~N} / \mathrm{m}$, nanosensors) [48]. All experiments in this study were operated under a narrow environment relatively humidity range $\mathrm{RH}=$ $21.4 \%-24.3 \%$ and basic constant temperature $23-24{ }^{\circ} \mathrm{C}$.

\section{Results and discussion}

In bimodal AFM, the analytical expressions of the rectangular cantilever beam vibration have been introduced $[49,50]$. From the equations of the bimodal motion, the second order flexural phase $\phi_{2}$, the first order torsional amplitude $A_{\mathrm{tr}}$, and torsional phase $\phi_{\text {tr }}$ at two resonance frequency peaks can be imaged in Fig. 2, where the matrix is PS and the circular area is LDPE, respectively. 


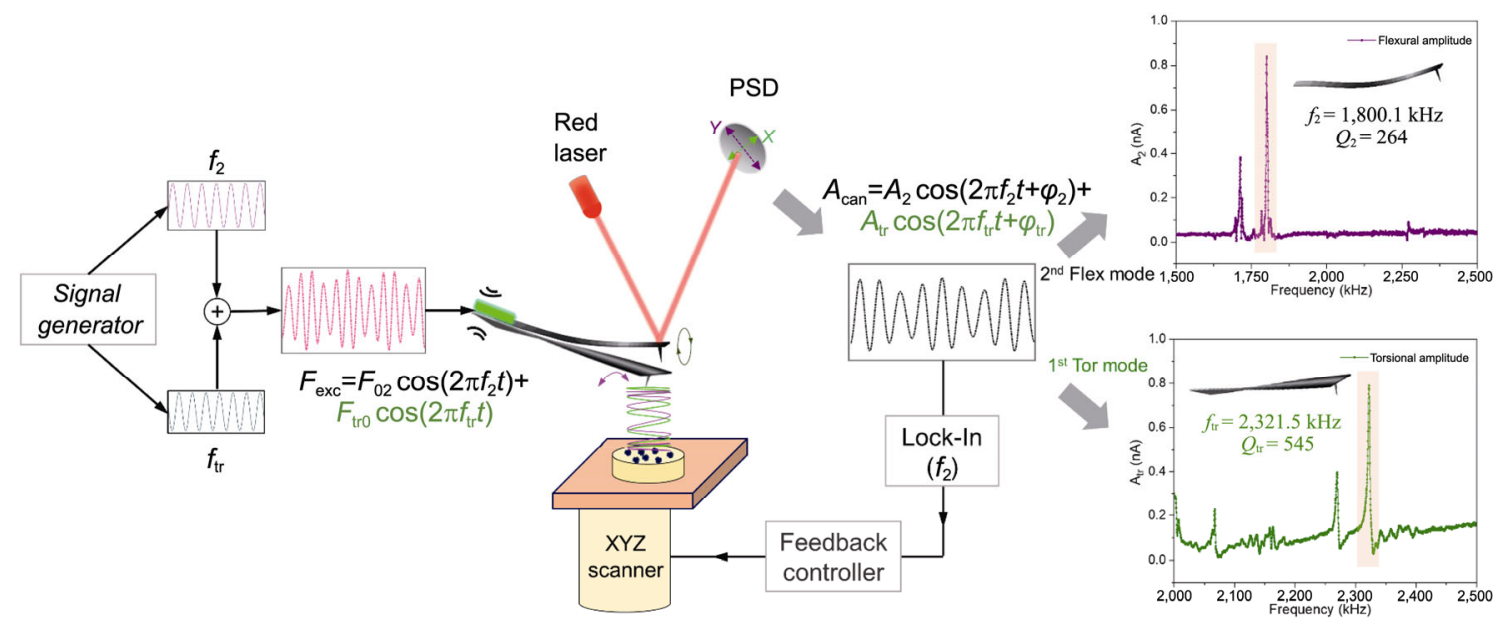

Fig. 1 Bimodal schematic description and control system, showing the bimodal driving signal contains the second order flexural and first order torsional mode frequencies, respectively. The second order flexural and the first order torsional vibration siganl spectra are shown in the purple and green curves, respectively. The flexural signal is used for topography feedback and the flexural dissipation, while the torsional signal is utilized to detect the torsional dissipation.

In a series of bimodal experiments, the different vibration modes in orthogonal directions were simultaneously excited by the sinusoidal voltage to detect the polymer blend of PS and LDPE. The second order free flexural amplitude $A_{02}$ were kept about $47 \mathrm{~nm}, 21 \mathrm{~nm}, 10 \mathrm{~nm}$, respectively. In addition, there is a new change rule of the energy dissipation power, and three different contact states can be distinguished by referring to the variation of the dissipation power and phase curves. The image contrast was greatly enhanced in the quasi-contact state. The friction energy dissipation coefficient was proposed to measure the relative magnitude of energy dissipation in both directions. Dynamic interaction and torsional vibration signals can be recorded in the repulsive force region, improving the edge detection capability.

\subsection{Bimodal phase images}

In Fig. 2, the bimodal phase images and curves under different $A_{02}$ in various setpoint ratio (0-1) are displayed with $A_{\mathrm{tr}}=3.16 \mathrm{~nm}$. The setpoint ratio abscissa $\left(A_{2} / A_{02}\right)$ refers to the operation amplitude $A_{2}$ as a percentage of the free resonance amplitude $A_{02}$ in the feedback. The larger the setpoint ratio is, the farther the tip is from the surface. When the setpoint ratio is zero, the tip contacts the sample surface and no oscillation can be sustained. The $90^{\circ}$ black dot line is the boundary between the attractive and repulsive interaction region [51]. As shown in Figs. 2(a) and 2(b), the histograms extracted from $\phi_{2}$ and $\phi_{\text {tr }}$ maps reveal the coexistence of two material components, where image contrast with $A_{02}=47 \mathrm{~nm}$ is larger than the other two with $A_{02}=21$ and $10 \mathrm{~nm}$ from the bimodal Gaussian peaks spacing. In Fig. 2(c), the second order flexural vibration is almost in the repulsive interaction region due to $\phi_{2}$ less than $90^{\circ}$. Only at high setpoint ratios and small $A_{02}$ can the flexural vibration work in the attractive interaction region. As $A_{02}$ decreases in Fig. 2(c), the setpoint ratio corresponding to $\phi_{2}=90^{\circ}$ lines also reduces. $\phi_{2}$ on LDPE is larger than that on PS with $A_{02}=47$ $\mathrm{nm}$, however, it is opposite with $A_{02}=10 \mathrm{~nm}$. And $\phi_{2}$ curve has an intersection at the setpoint around 0.36 with $A_{02}=21 \mathrm{~nm}$. Details show that the $\phi_{2}$ difference between the two components is not constant, but varies with $A_{02}$ and the setpoint ratio. It is influenced not only by the characteristics of the two materials, but also by the initial amplitude and the setpoint ratio, which should be taken into account when identifying the two materials. In Fig. $2(\mathrm{~d})$, the torsional vibration works in the repulsive state, because $\phi_{\text {tr }}$ is all below the $90^{\circ}$ black dot line.

\subsection{Dynamic friction dissipation and contact states}

For the case of large amplitudes used in bimodal 
(a)

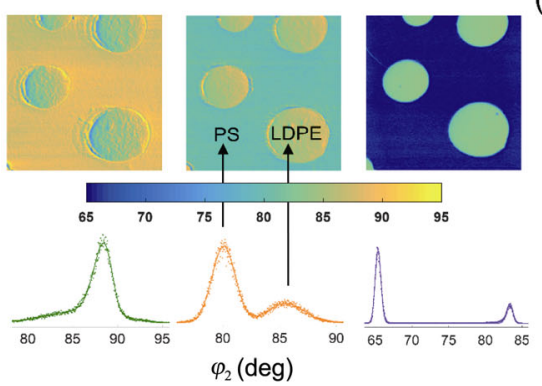

(c)

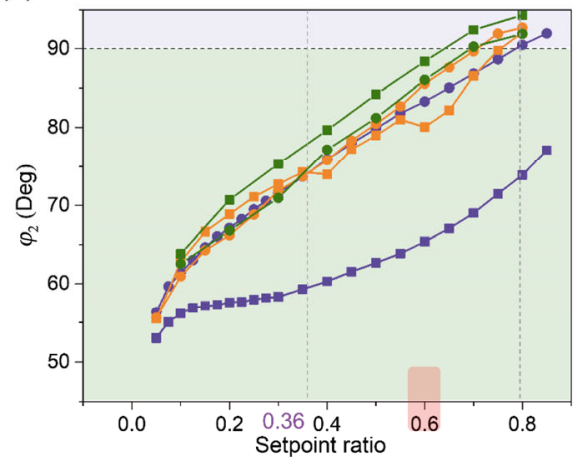

(b)

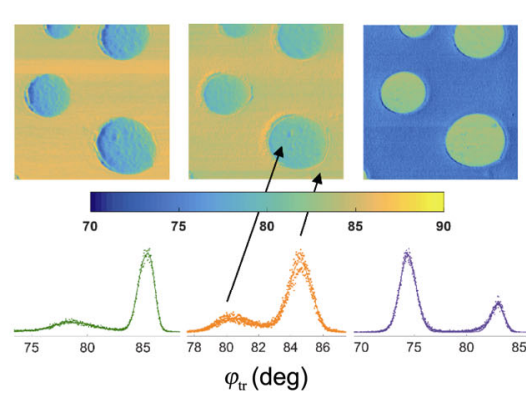

(d)

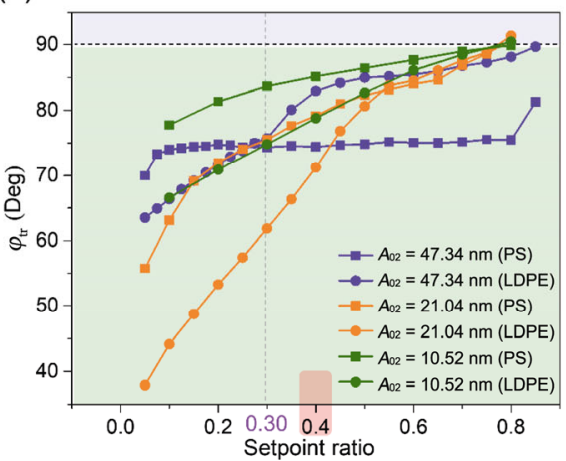

Fig. 2 The bimodal phase images $(10 \mu \mathrm{m} \times 10 \mu \mathrm{m})$ and curves under different $A_{02}$ in setpoint ratio range (0-1). (a) $\varphi_{2}$ images at setpoint ratio 0.6 and (b) $\varphi_{\text {tr }}$ images at setpoint ratio 0.4 with three $A_{02}$. (c) $\varphi_{2}$ and (d) $\varphi_{\text {tr }}$ curves. $A_{02}=47 \mathrm{~nm}$ (purple line), $A_{02}=21 \mathrm{~nm}$ (orange line), and $A_{02}=10 \mathrm{~nm}$ (green line). The solid square dots and circle dots represent the data on the PS and LDPE, respectively. The maximum torsional amplitude $A_{\mathrm{tr}}$ is $3.16 \mathrm{~nm}$.

AFM, the strength of the dissipative tip-sample interaction is different occurring during one cycle of oscillation. Qualitatively, the dissipative tip-sample interactions should have an appreciable value only close to the lower turnaround point of the oscillation cycle in tapping mode, while the viscous cantilever damping in air is proportional to the velocity, i.e., maximal at the average tip-sample position.

In the interaction region, the dissipative force between the tip and sample is the convolutions of the interactions with the velocity. According to the law of energy conservation, the dissipative tipsample interaction is in the balance of the power and it is the difference between the input energy and the eigen dissipation energy of the tip. In bimodal AFM, the average bimodal dissipation power $P_{\text {disnor }}$ and $P_{\text {distor }}$ per cycle can be derived by the following analytical expressions [52-55]:

$$
\begin{gathered}
P_{\text {disnor }}=\frac{\pi f_{2} k_{2} A_{2} A_{02}}{Q_{2}}\left(\sin \varphi_{2}-\frac{A_{2}}{A_{02}}\right) \\
P_{\text {distor }}=\frac{\pi f_{\mathrm{tr}} k_{\mathrm{tr}} A_{\mathrm{tr}} A_{0 \mathrm{tr}}}{Q_{\mathrm{tr}}}\left(\sin \varphi_{\mathrm{tr}}-\frac{A_{\mathrm{tr}}}{A_{0 \mathrm{tr}}}\right)
\end{gathered}
$$

where $k_{2}, f_{2}, \varphi_{2}$, and $Q_{2}$ are the second order mode stiffness, resonance frequency, phase and quality factor, respectively. $A_{2}$ and $A_{02}$ are the second order flexural operation and free amplitude of the cantilever. $P_{\text {disnor }}$ and $P_{\text {distor }}$ are the flexural and friction tip-sample energy dissipation power, respectively. The suffix tr refers to the first order torsional vibration mode.

As shown in Figs. 3(a) and 3(b), the $P_{\text {disnor }}$ and $P_{\text {distor }}$ values are mapped at different setpoint ratio $0.2,0.5$, and 0.7 with $A_{02}=47 \mathrm{~nm}$. Overall, $P_{\text {distor }}$ is about a few thousandths to a few percent of $P_{\text {disnor }}$ from the color bar. The dissipation power data values in Figs. 3(c) and 3(d) were extracted from the Gaussian bimodal peaks in the histograms. The $P_{\text {disnor }}$ curve shows a parabolic trend, and the maximum is around setpoint ratio 0.5 in Fig. 3(c), indicating that the dominant interaction contributions are the long range surface adhesion and normal viscoelasticity [56]. $P_{\text {disnor }}$ on LDPE is larger than that on PS, especially with $A_{02}=47 \mathrm{~nm}$. Large $A_{02}$ can exert relatively strong force on the surface of low modulus specimens, such as LDPE, resulting 
in high flexural energy dissipation. When the tip is touching the sample surface, it is harder for PS to produce the large deformation and energy dissipation compared with LDPE. It seems that greater amplitudes cause contact or some penetration into the sample, which in turn produces greater repulsive forces. In Fig. 3(d), $P_{\text {distor }}$ curves also show the parabolic trends, but the vertexes are not in the same place, mainly due to the closed-loop flexural vibration and open-loop torsional vibration. $A_{\text {tr }}$ is different on PS and LDPE in Fig. 5(c), but $A_{2}$ is the setpoint operation amplitude for the close-loop stable imaging, indicating $A_{2}$ maintains constant when scanning the sample. $P_{\text {distor }}$ reaches the maximum in the intermediate setpoint ratio region, whereas decreases on the other two sides. As $A_{02}$ decreases, $P_{\text {distor }}$ is closer to $P_{\text {disnor, }}$ indicating the torsional vibration only takes effect in a short period of the total vibration cycle when close to the sample surface, whereas during most of the period, the tip produces weak interaction farther away. From the shapes of the torsional dissipation power curves, the torsional interaction is dominated by the long range surface adhesion and lateral viscoelasticity [57]. If the tip is drawn into the surface, the torsional friction energy dissipation power will suddenly increase due to the deep pressure and the indentation [40]. In Fig. 3(d), $P_{\text {distor }}$ decreases slightly near the sample surface, showing the tip overcomes the attractive force by the vibrating. The stable vibration near the sample surface keeps the tip small amplitude. Besides, in the bimodal AFM experiments with the second order flexural vibration as feedback, the near surface $P_{\text {distor }}$ can be detected, which is caused by the dynamic lateral force or friction.

Three different contact states can be distinguished through the process of phase and energy dissipation curves in Figs. 2(c) and 3(c). When the phase is higher than $90^{\circ}$, the interaction is in the attractive region, which can be considered as the noncontact state. When the phase value is less than $90^{\circ}$, the interaction is in the repulsive region, which can be considered as the contact state. But there is another (a)

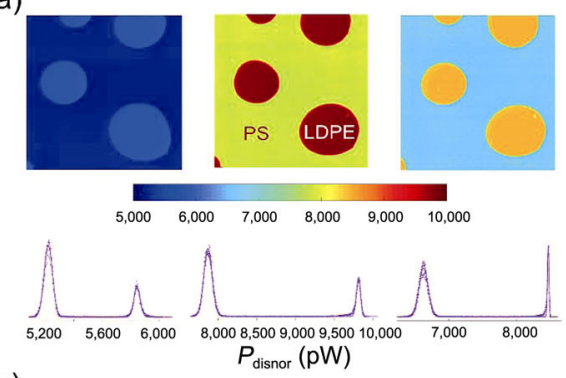

(c)

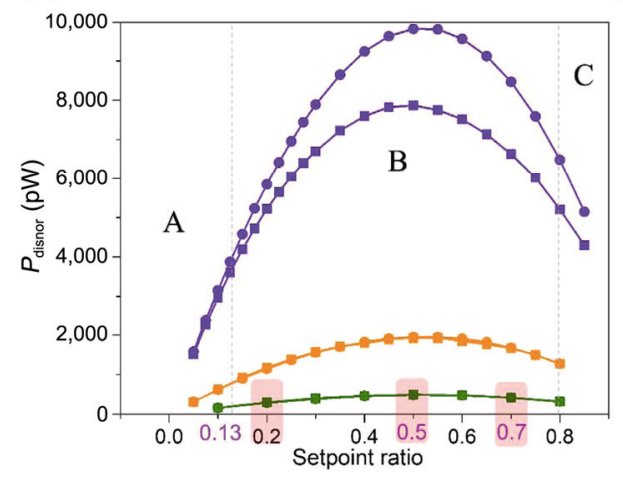

(b)

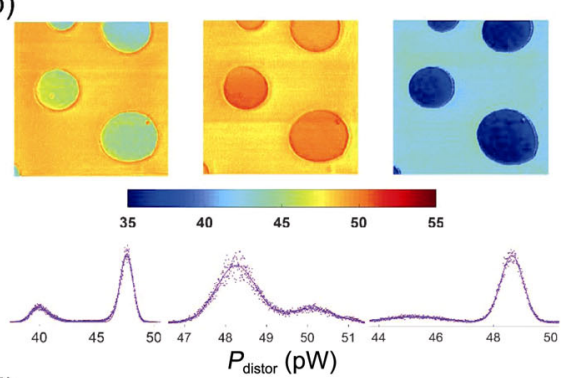

(d)

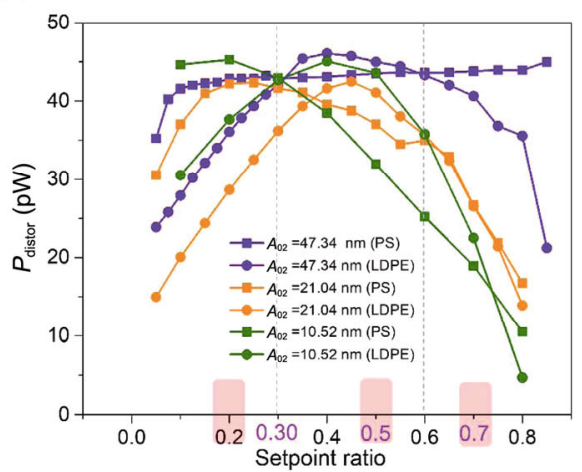

Fig. 3 The energy dissipation power maps $(10 \mu \mathrm{m} \times 10 \mu \mathrm{m})$ and curves under different $A_{02}$ in setpoint ratio range (0-1). (a) $P_{\text {disnor }}$ and (b) $P_{\text {distor }}$ images at setpoint ratio $0.2,0.5$, and 0.7 with $A_{02}=47 \mathrm{~nm}$. (c) $P_{\text {disnor }}$ and (d) $P_{\text {distor }}$ curves. Part A, part B, and part $\mathrm{C}$ refer to the contact, quasi-contact, and noncontact state, respectively. $A_{02}=47 \mathrm{~nm}$ (purple line), $A_{02}=21 \mathrm{~nm}$ (orange line), and $A_{02}=10 \mathrm{~nm}$ (green line). The solid square dots and circle dots represent the data on the PS and LDPE, respectively. The maximum torsional amplitude $A_{\mathrm{tr}}$ is $3.16 \mathrm{~nm}$. 
state, called quasi-contact state, a transition between contact and noncontact state. In this state, there are coexistences of attractive and repulsive interactions, corresponding to the position where the phase is equal to $90^{\circ}$, which is only a fleeting operating setpoint. For the general micro cantilever with low force constant, it is in the unstable working state. In this work, the effective second order flexural force constant is about $844.1 \mathrm{~N} / \mathrm{m}$. Even in the quasi-contact state, the phase signal will not change dramatically. On LDPE component with $A_{02}=47$ $\mathrm{nm}$, from the remote resonance state to the $90^{\circ}$ phase of the micro cantilever, the total attraction, including the long range dissipative interfacial force, is small and meanwhile the repulsive force is negligible from the beginning. As shown in part C of Fig. 3(c), the energy dissipation of the whole system is in a low state, considered as the noncontact state. With the decrease of the setpoint ratio, it is suggested that from the $90^{\circ}$ phase point (setpoint 0.8 ) to the setpoint 0.13 of the flexural dissipation curve may be the quasi-contact state, exhibited in the part B of Fig. 3(c). The given amplitude reduction involves tip-sample mechanical contact, especially when using a cantilever with large force constant and compliant materials [58]. However, at the beginning of approach, the average contact force is extremely weak due to the short contact time and the tiny deformation. As the repulsive force increases gradually, the total energy dissipation increases when the phase curve is less than $90^{\circ}$. At the same time, the long range dissipative interfacial forces, the short range surface adhesion hysteresis, and the viscoelastic force all increase [51]. The flexural vibration signal has the maximum contrast. From setpoint 0.13 to 0 , as shown in the part A of Fig. 3(c), due to the decrease of the viscoelastic force, the total energy dissipation is reduced. At this time, it is considered as the contact state, and the contact time between the tip and the sample becomes longer. The amplitude and vibration speed decrease, and the sample deformation and its rate are small consequently a reduced viscoelastic force.

Similar to the friction coefficient defined as the ratio of friction to normal force under static contact, friction dissipation coefficient $\eta$ can be defined as the ratio of torsional $P_{\text {distor }}$ to normal $P_{\text {disnor }}$ at different distances. The parameter varies with the setpoint ratio and represents the percentage of friction dissipation in the normal energy dissipation power, which is used to measure the relative magnitude of energy dissipation in both directions. The analytical expression is as follows:

$$
\eta=\frac{P_{\text {distor }}}{P_{\text {disnor }}}
$$

The friction dissipation coefficient can not be compared on one scale under different free amplitudes, so it is necessary to normalize the coefficient. Normalized friction dissipation coefficient $\eta^{*}$ is defined as the ratio of each point value $\eta$ to the maximum $\eta_{\max }$.

$$
\eta^{*}=\frac{\eta}{\eta_{\max }}
$$

The normalized friction dissipation coefficient includes two types in Fig. 4. One is $\eta_{1}{ }^{*}$ of the first order vibration feedback, the other is $\eta_{2}{ }^{*}$ of the second order vibration feedback. The normalized friction dissipation coefficient ranges from 0 to 1 , and the abscissa is the setpoint ratio.

In the previous study, with the first order free flexural vibration amplitude $A_{01}=254 \mathrm{~nm}$, the first order normalized friction dissipation coefficient 0.15 and 0.5 are the cut-off points of noncontact region III $\left(\eta_{1}{ }^{*}<0.15\right)$, quasi-contact region II $\left(0.15<\eta_{1}{ }^{*}<\right.$ $0.5)$, and contact state region I $\left(0.5<\eta_{1}{ }^{*}\right)$ [40]. A slight mutation occurs at the setpoint ratio 0.14 with $A_{01}=123$ and $65 \mathrm{~nm}$, where the quasi-contact and contatct states can be distinguished obviously in Figs. 4(b) and 4(c). It is also the transitional setpoint at the flexural phase $90^{\circ}$ boundary. The corresponding normalized friction dissipation coefficients are 0.16 and 0.15 , respectively. The cut-off points basically coincide on PS and PLDE in Figs. 4(a)-4(c), whereas it shows a big difference in Figs. 4(d)-4(f), implying the second order vibration feedback affects the energy dissipation ratio distribution. $\eta_{2}{ }^{*}$ on LDPE is greater than that on PS, indicating the friction dissipation of low elasticity modulus specimens is larger. The regions I-III in Fig. 4(d) correspond to the regions A-C in 

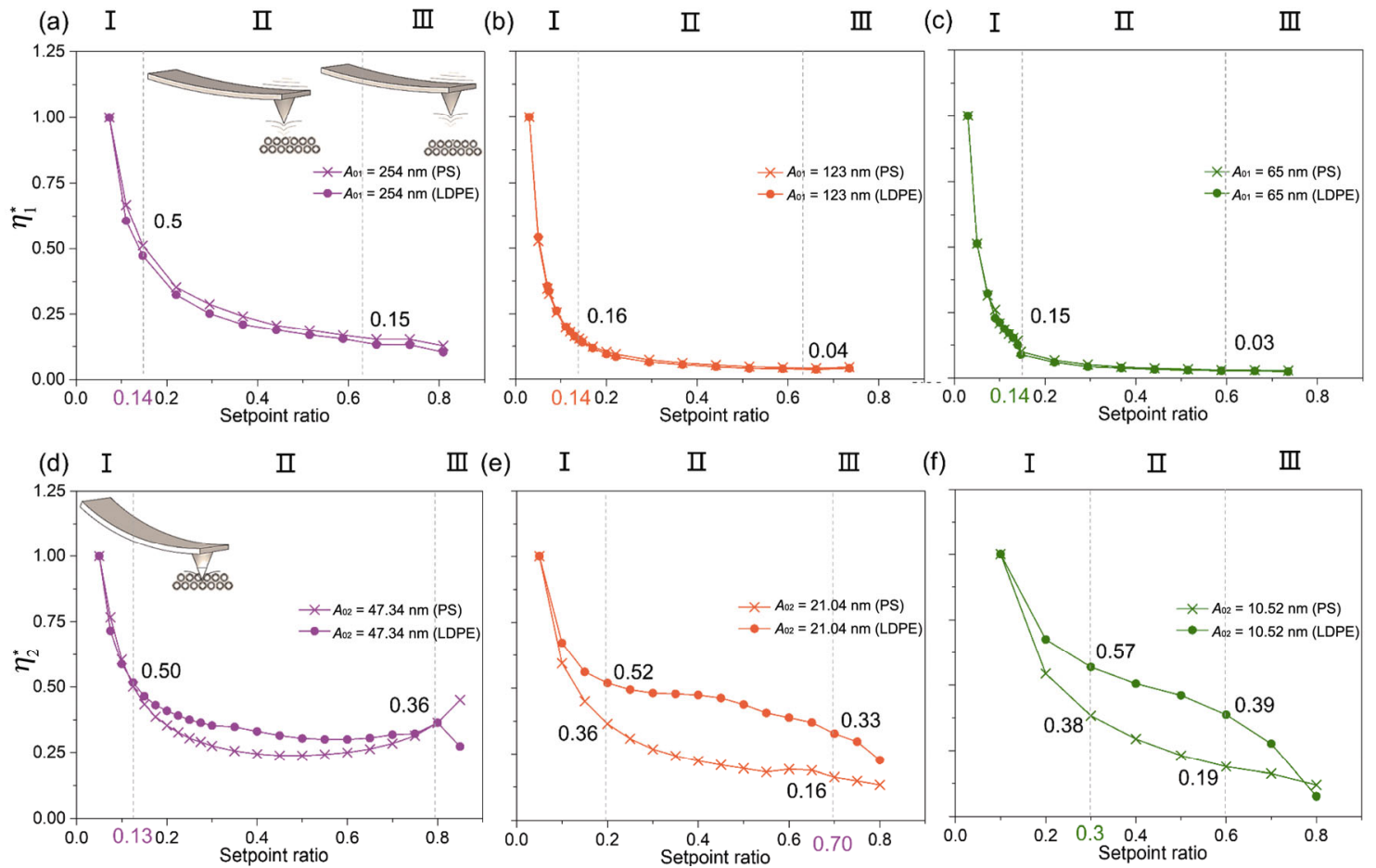

Fig. 4 Normalized friction dissipation coefficient at different setpoint ratios. (a)-(c) $\eta_{1}{ }^{*}$ with the first order flexural vibration feedback. The original data are from Ref. [39]; (d)-(f) $\eta_{2}{ }^{*}$ with the second order flexural vibration feedback. The cross dot line represents PS and the circle dot line represents the LDPE component. The purple, orange, and green lines mean the different free amplitudes. The tip-sample illustrations depict the noncontact and quasi-contact states in (a)-III and (a)- II, respectively. The illustration in (d)- I depicts the contact state.

Fig. 3(c). Unlike the first order vibration feedback, the distinction between the quasi-contact and contact regions in Figs. 4(d)-4(f) is not very clear. The cut-off points of noncontact and quasi-contact states are distinguished by the $90^{\circ}$ phase lines in Fig. 2(c). In the noncontact region III, the normalized friction dissipation coefficients are very small and the slope is basically unchanged, leading to the poor phase contrast due to the weak force. In the quasi-contact region II, the coefficients increase slightly and the phase contrast maintains the maximum, especially with the first order flexural vibration feedback, mainly because the coexistence of the contact and noncontact states enhance the interaction. In the contact region I, the friction dissipation coefficient increases rapidly to 1 , and the high contact force reduces the flexural phase image contrast instead.

There is a famous argument proposed by Israelachvili that the magnitude of friction is closely related to the strength of the normal adhesion hysteresis, which in fact is the relationship between the flexural dissipation and the lateral dissipation [59]. The microscopic friction is mainly controlled by adhesion and load. Under ideal conditions and low load, friction mainly comes from the adhesion control part. Therefore, the friction energy conversion coefficient $\varepsilon$ of the viscous hysteresis energy dissipation can be defined.

In the tapping mode at high amplitude setpoint ratios, the average load is small. It can be seen from the friction dissipation coefficient curves that the coefficient changes little in the noncontact and quasi-contact states, indicating that the two kinds of dissipation maintain a certain proportional relationship and correlation. It can be concluded that the energy conversion ratio is basically constant in these two states, mainly because the surface energy asymmetry $\left(\gamma_{\mathrm{r}}-\gamma_{\mathrm{a}}\right)$ of the tip is basically unchanged during the process of approaching or withdrawing contact, which is very similar to the description of adhesion control friction in the monograph [59]. However, the friction dissipation coefficient increases rapidly in the contact state, 
indicating that the influence of flexural dissipation on friction is already very weak, as the contact load increases sharply and the lateral friction is partly derived from the load control component, enlarging the overall friction energy dissipation. The friction dissipation coefficient of the three contact states is similar to the friction energy conversion ratio of the viscous hysteresis energy dissipation, which not only proves the rationality of the statement experimentally, but also expands its applications.

\subsection{Near imaging and enhanced contrast}

In bimodal AFM, heterogeneous polymers images show the contrast of two different materials. Sometimes, the contrast cannot be visually observed, so the quantitative study is necessary. The image pixels can be divided into two parts as the frequency distribution histograms, which are fitted by Gaussian function. The normalized histograms and the bimodal distribution function are fitted by [27]:

$$
f(\varphi)=\frac{p}{\sigma_{1} \sqrt{2 \pi}} \mathrm{e}^{-\frac{\left(\varphi-\mu_{1}\right)^{2}}{2 \sigma_{1}^{2}}}+\frac{1-p}{\sigma_{2} \sqrt{2 \pi}} \mathrm{e}^{-\frac{\left(\varphi-\mu_{2}\right)^{2}}{2 \sigma_{2}^{2}}}
$$

where $\mu_{1}$ and $\mu_{2}$ are the normal distribution means of two components, $\sigma_{1}$ and $\sigma_{2}$ are the standard deviations, $p$ is the ratio of pixels in the first distribution, and (1-p) the ratio of pixels in the second. The means and standard deviations were used to define the average and quantify the variation or dispersion of a set of phase values $(0<$ $\left.\varphi<180^{\circ}\right)$. Ashman's $D$ refers to the phase and amplitude image contrast, and $D>2$ is a necessary condition to distinguish two mixed materials. $D$ can be derived by the following statistical formula [60]:

$$
D=\frac{\sqrt{2}\left|\mu_{1}-\mu_{2}\right|}{\sqrt{\sigma_{1}^{2}+\sigma_{2}^{2}}}
$$

The $\varphi_{2}$ contrast, $\varphi_{\text {tr }}$ contrast, $A_{\text {tr }}$ and its contrast under different $A_{02}$ in setpoint ratio range (0-1) are shown in Fig. 5. The setpoint axis is divided into three sections I, II, and III by the variation trend of contrast. As for $\varphi_{2}$ contrast in Fig. 5(a), the contrast with $A_{02}=47 \mathrm{~nm}$ is much higher than the other two contrast lower than 2. The effect of increasing free amplitude $A_{02}$ at a fixed setpoint ratio is similar to that of increasing setpoint ratio at a fixed free amplitude, both of which lead to strong interaction. The velocity-dependent viscous damping increases with the free amplitude and the setpoint ratio. In the process, the tip velocity and tapping force increase, enhancing the effect of viscoelasticity [51, 56]. Therefore, for a given setpoint, the phase contrast is higher at the large free amplitude. The $\varphi_{2}$ contrast is obviously higher than the $\varphi_{1}$ contrast in the previous study using the first order flexural vibration mode as the feedback, especially with large free amplitude [40]. The $A_{\text {tr }}$ and $\varphi_{\text {tr }}$ contrast are essentially affected by the in-plane (lateral) heterogeneity of the surface. In Fig. 5(b), for the purple curve with high $A_{02}$, the $\varphi_{\text {tr }}$ contrast reaches the minimum at a setpoint ratio around 0.28 . The $\varphi_{2}$ contrast in Fig. 5(a) is larger than the $\varphi_{\text {tr }}$ contrast in Fig. 5(b) with $A_{02}=47$ $\mathrm{nm}$, however in the orange and green curves with $A_{02}=21 \mathrm{~nm}$ and $10 \mathrm{~nm}$ in Figs. 5(b) and 5(d), the ability of torsional vibration to distinguish different component materials in the close scanning distance is pretty better. Because the torsional vibration is in the repulsive interaction region, the dynamic lateral force plays an important role in the amplitude reduction. The amplitude decreases as the setpoint ratio reduces, namely, the tip is approaching the sample surface. The $A_{\text {tr }}$ contrast region is separated by the black dot line $D=2$ in Fig. 5(d), showing a similar trend with $\varphi_{\text {tr }}$ contrast curves. The setpoint ratio intersection 0.32 in $A_{\text {tr }}$ curves of Fig. 5(c) is related to the minimum in Figs. $5(\mathrm{~b})$ and $5(\mathrm{~d})$. However, within low setpoint ratio region I (0-0.13), the $\varphi_{2}$ contrast decreases but $\varphi_{\text {tr }}$ and $A_{\text {tr }}$ contrast increase, indicating the torsional vibration is sensitive to the near surface scanning. At this time, the dynamic behaviors of the tip change greatly, and the tip may be absorbed on the sample surface. From the above analyses, in order to obtain better contrast between the two materials, the best method is to compare the phase and torsional amplitude within the intermediate setpoint region II (0.13-0.8), where both contact and noncontact states exist. The special contact state can be defined 

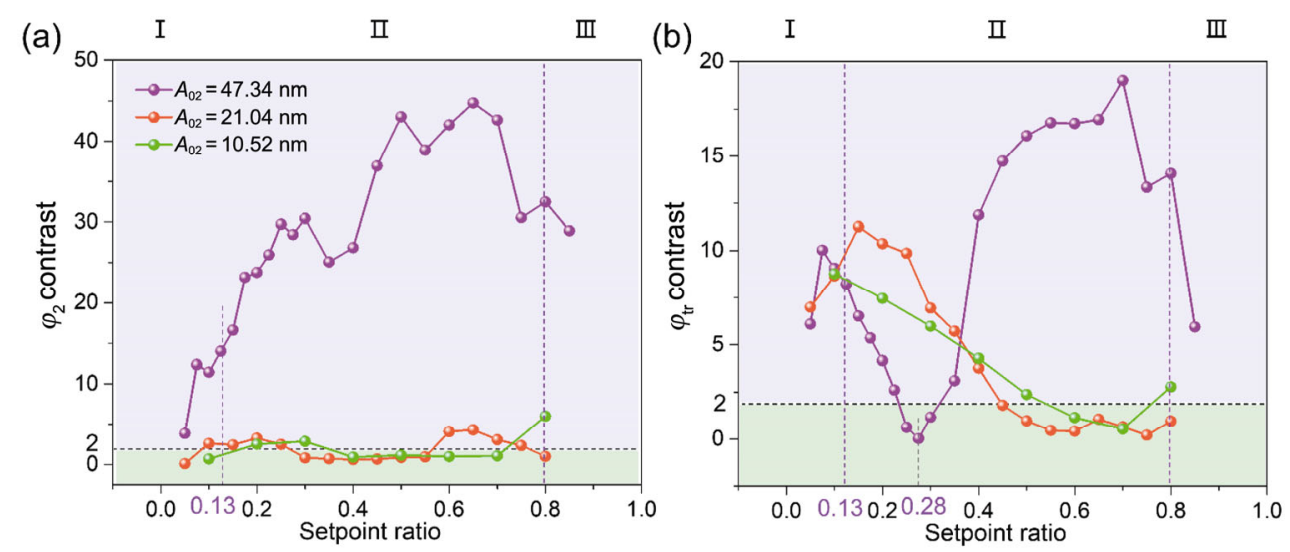

(c)

(d)
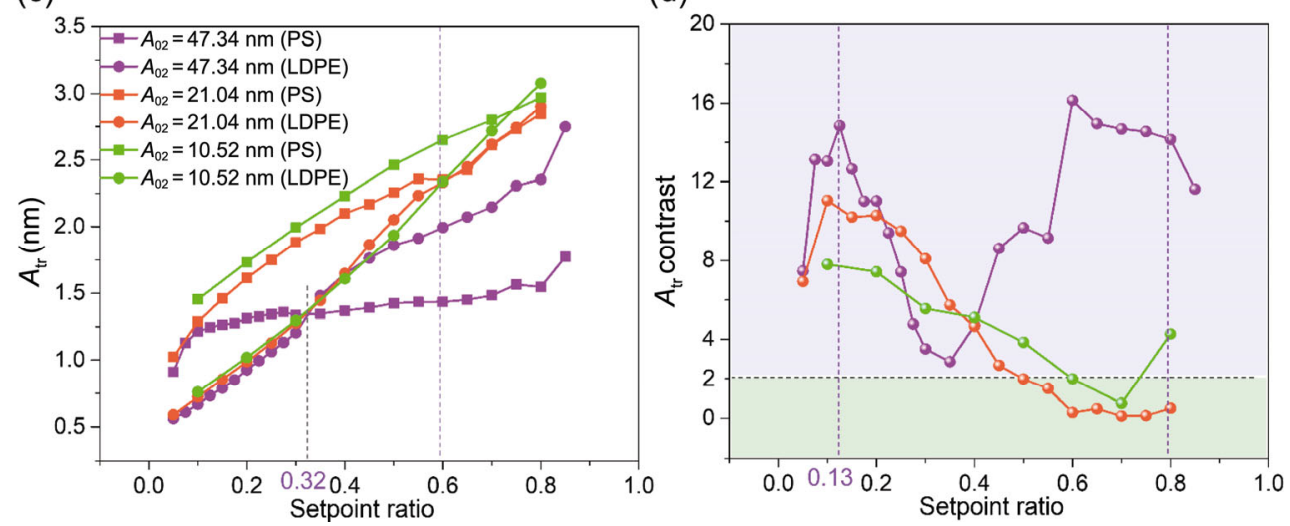

Fig. 5 The amplitude, phase and contrast of the second order flexural vibration and the first order torsional vibration under different $A_{02}$ in setpoint ratio range (0-1). The setpoint ratio axis is divided into three regions I, II and III. (a) $\varphi_{2}$ and (b) $\varphi_{\mathrm{tr}}$ contrast. (c) $A_{\text {tr }}$ and (d) its contrast. The purple dotted lines are the boundaries between different contrast stages. $A_{02}=47 \mathrm{~nm}$ (purple line), $21 \mathrm{~nm}$ (orange line), and $10 \mathrm{~nm}$ (green line). The solid square dots and circle dots represent the data on the PS and LDPE, respectively.

as the quasi-contact state, in which the phase and amplitude contrast can be greatly enhanced due to the sufficient interaction between the tip and the sample. However, the $\varphi_{2}$ contrast is low with $A_{02}=$ 21 and $10 \mathrm{~nm}$. In this situation, the image contrast depends more on torsional signals. Also, compared with the situation using the first order flexural vibration feedback, the $\varphi_{\mathrm{tr}}$ contrast and $A_{\text {tr }}$ contrast are larger, especially in the close scanning range with small free amplitude [40]. Within high setpoint ratio region III (0.8-1), as the energy dissipation reduces, the $\varphi_{2}, A_{\mathrm{tr}}$, and $\varphi_{\mathrm{tr}}$ contrast decrease.

Under atmosphere environmental conditions, a thin layer of water may be absorbed on the sample surface, resulting a meniscus or liquid bridge at close distances between the tip and the surface [61]. The attractive interaction contains the capillary force, Van der Waals' force and the electrostatic force, causing the unsteady motion that the tip is drawn into the sample surface. They all increase with the distance decrease. Therefore, it is important to note at what distance the pull-in phenomenon occurs. A useful explanation is that when the kinetic energy of the tip vibration and the potential energy of its position are less than the energy of the attractive field on the sample surface, the tip will be pulled in, and the distance at this time is the critical value [62]. Similar phenomena have been observed in static force-distance curves. Therefore, increasing the kinetic energy of tip vibration is an effective way to avoid the pull-in phenomenon. The simplest way is to replace the cantilever beam with high force constant (e.g., Q-plus force sensor) or to excite high-order vibration [25]. In this experiment, the goal is achieved by exciting the higher flexural vibration mode.

When the tip is closely scanning the sample surface, the image quality is poor usually at the 
first order flexural vibration feedback [40]. However, when the second order flexural vibration is used as the feedback, the spatial resolution can be improved [29]. The bimodal mode, including the torsional vibration, is a breakthrough point for measuring dynamic friction effects at close range. The setpoint ratio is 0.1 in both experiments to determine the near scanning to the same extent compared with different free amplitudes. From the left images in Figs. 6(a)-1 and 6(b)-1, we can see the phase contrast is low, especially at the edge of the heterogeneous materials. Only the torsional amplitude can clearly show the different components of the materials in Fig. 6(c)-1. When scanning close to the sample surface, the tip can be drawn into the substrate by strong attractive forces from the first order vibration feedback loop. At this time, the phase signal fails to distinguish the materials except for the torsional amplitude. When employing the second flexural vibration as the feedback, under $A_{02}=47 \mathrm{~nm}$, the heterogeneous polymers are well distinguished by the second order flexural phase $\varphi_{2}$ and the first order torsional phase $\varphi_{t r}$ and amplitude $A_{t r}$ in Fig. 6-2. For smaller free amplitude $A_{02}$ in Figs. 6-3 and 6-4, there are some fine structures in the boundary between the two materials, because the decrease of the tip-sample distance can lead to strong interaction in the lateral direction. The flexural vibration of the tip reflects the out-of-plane sample nano-mechanical properties, while the lateral vibration of the tip is sensitive to the in-plane forces, or called dynamic friction force. The torsional phase and amplitude signals have more advantages in detecting micro structures, especially the edge of heterogeneous materials in Figs. 6(b)-4 and 6(c)-4. The sample can deform laterally, resulting in strong friction interaction.

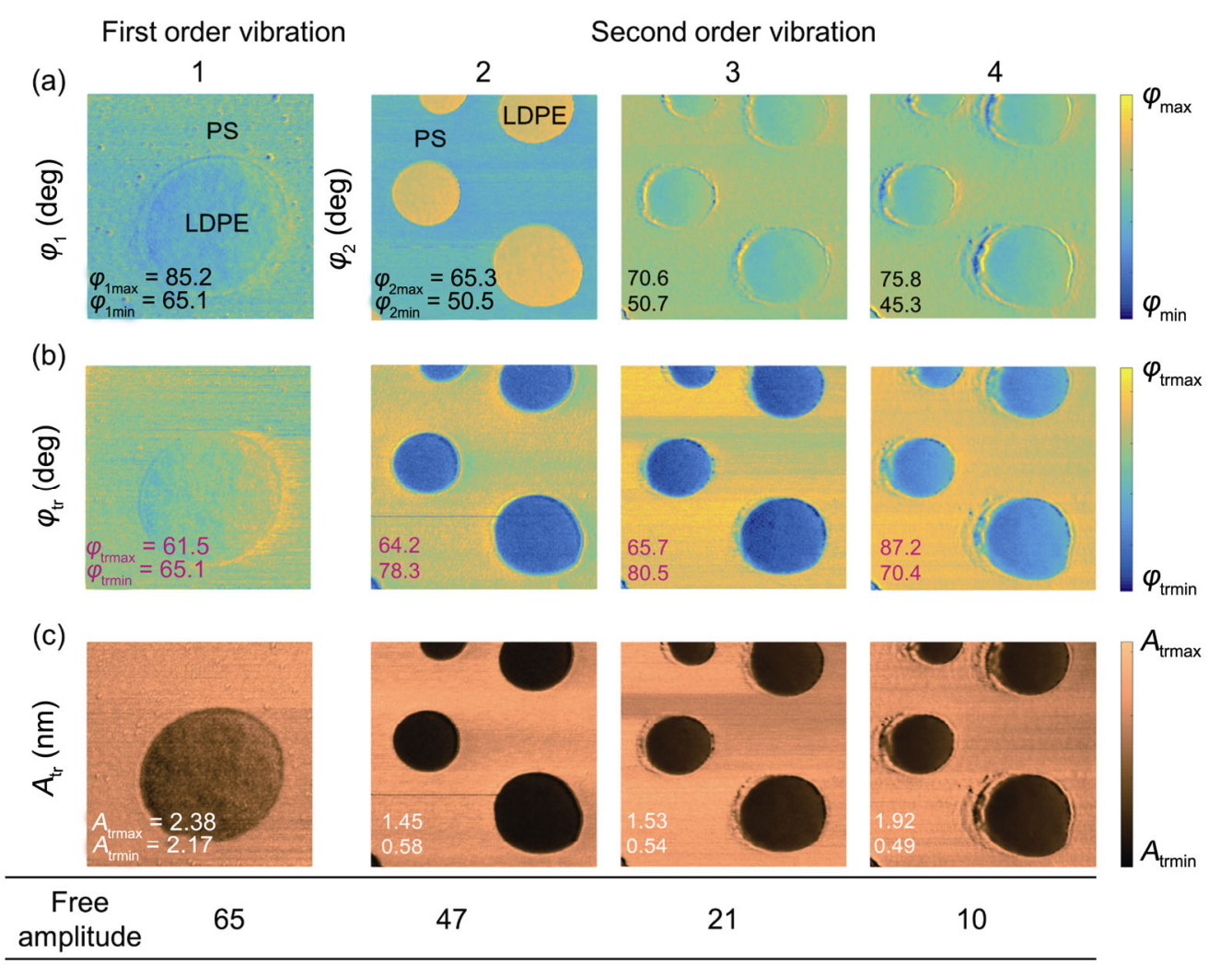

Fig. 6 The bimodal AFM scanning images of PS-LDPE at 0.1 setpoint ratio when free amplitude $A_{01}=65 \mathrm{~nm}, A_{02}=47 \mathrm{~nm}, 21$ $\mathrm{nm}$ and $10 \mathrm{~nm}$, respectively. (a) The first and second order flexural vibration phase $\varphi_{1}$ and $\varphi_{2}$. (b) The first order torsional vibration phase $\varphi_{t r}$ and (c) the first order torsional vibration amplitude $A_{t r}$. The images $(4.5 \mu \mathrm{m} \times 4.5 \mu \mathrm{m})$ in the first column on the left are obtained by the first order flexural vibration feedback, while the images $(10 \mu \mathrm{m} \times 10 \mu \mathrm{m})$ in the second to fourth columns on the right are obtained by the second order flexural vibration feedback. Numbers in bottom left corners show the limits of the color scales $\varphi_{\max }, \varphi_{\min }$ and $A_{\text {trmax }}, A_{\text {trmin }}$ for each amplitude and phase image respectively. 


\section{Conclusions}

In the present work, a bimodal AFM technique that combines the normal and lateral tip motion by simultaneously driving the cantilever at both the second order flexural and the first order torsional eigenmodes was developed. It can provide valuable insights into the out-of-plane and in-plane dissipation power of the polymer blend of PS and LDPE, which are not accessible by standard AFM methods. The inplane dissipation is much smaller than the out-ofplane dissipation at three free flexural amplitudes $A_{02}$. In the intermediate setpoint ratio region, the contrast of the compliant heterogeneous materials was enhanced. Besides, the pull-in problem due to strong attractive force near the sample surface can be addressed by exciting the second order flexural vibration mode. The edges of the heterogeneous materials are clearer in the second order flexural vibration feedback loop than that in the first order, indicating the high order vibration improves the resolution and image contrast. The friction dissipation coefficient was proposed and three different contact states were determined from the energy dissipation and phase curves. The image contrast was greatly enhanced in the quasi-contact state where the attractive and repulsive interactions coexists. The quasi-contact state is of great significance to the research of friction.

To better understand the origination of friction in the microscale, the normal and torsional energy dissipation between the tip and sample surfaces several nanometers apart are discussed. It is found that the relationship between normal distance and friction energy dissipation is not linear, but parabolic. From the point of view of contact friction, it is generally believed that the closer the distance is, the stronger the force is and the greater the friction energy dissipation power is. However, for the dynamic AFM mode, the closer the distance is, the force will change from attraction to repulsion, and reduced amplitude will lead to the decrease of the system energy, thus reducing the friction dissipation. Friction originates from two relatively moving surfaces in a force field, regardless of the form of motion. In the micro-nano world, the flexural vibration and torsional vibration are similar to some extent. Therefore, the energy dissipation verse distance change regularities in bimodal directions are both similar to parabolic trend, which may help to understand the modulation effect of vibration on friction. This work opens the window from noncontact to contact friction, which provides the possibility for the study of friction in intermittent contact state, and provides a way to understand the actual friction conditions and energy dissipation in the future. This work demonstrates that bimodal technique is an effective method for capturing the friction dissipation and high resolution images, which is a good complement to the existing methods such as contact mode AFM or other contact friction and wear measuring instruments.

\section{Acknowledgements}

This research is financially supported by the National Natural Science Foundation of China (No. 51527901).

Open Access This article is licensed under a Creative Commons Attribution 4.0 International License, which permits use, sharing, adaptation, distribution and reproduction in any medium or format, as long as you give appropriate credit to the original author(s) and the source, provide a link to the Creative Commons licence, and indicate if changes were made.

The images or other third party material in this article are included in the article's Creative Commons licence, unless indicated otherwise in a credit line to the material. If material is not included in the article's Creative Commons licence and your intended use is not permitted by statutory regulation or exceeds the permitted use, you will need to obtain permission directly from the copyright holder.

To view a copy of this licence, visit http://creativecommons.org/licenses/by/4.0/.

\section{References}

[1] Urbakh M, Meyer E. NANOTRIBOLOGY The renaissance of friction. Nat Mater 9(1): 8-10 (2010)

[2] Kisiel M, Gnecco E, Gysin U, Marot L, Rast S, Meyer E. Suppression of electronic friction on $\mathrm{Nb}$ films in the 
superconducting state. Nat Mater 10(2): 119-122 (2011)

[3] Holmberg K, Erdemir A. Influence of tribology on global energy consumption, costs and emissions. Friction 5(3): 263-284 (2017)

[4] Tan X, Luo J. Research Advances of Lubrication. China Mechanical Engineering 31(2): 145-174, 189 (2020)

[5] Meng Y, Xu J, Jin Z, Prakash B, Hu Y. A review of recent advances in tribology. Friction 8(2): 221-300 (2020)

[6] Binnig G, Quate C F, Gerber C. Atomic force microscope. Phys Rev Lett 56(9): 930-933 (1986)

[7] Albrecht T R, Grutter P, Horne D, Rugar D. Frequency-modulation detection using high-Q cantilevers for enhanced force microscope sensitivity. J Appl Phys 69(2): 668-673 (1991)

[8] Giessibl F J. Atomic resolution of the silicon (111)-(7x7) surface by atomic force microscopy. Science 267(5194): 68-71 (1995)

[9] Herruzo E T, Asakawa H, Fukuma T, Garcia R. Three-dimensional quantitative force maps in liquid with 10 piconewton, angstrom and sub-minute resolutions. Nanoscale 5(7): 2678-2685 (2013)

[10] Martin-Jimenez D, Chacon E, Tarazona P, Garcia R. Atomically resolved three-dimensional structures of electrolyte aqueous solutions near a solid surface. Nat Commun 7: 12164 (2016)

[11] Ky D L C, Khac B C T, Le C T, Kim Y S, Chung K H. Friction characteristics of mechanically exfoliated and CVD-grown single-layer $\mathrm{MoS}_{2}$. Friction 6(4): 395-406 (2018)

[12] Luo J, Zhou X. Superlubricitive engineering-Future industry nearly getting rid of wear and frictional energy consumption. Friction 8(4): 643-665 (2020)

[13] Mate C M, McClelland G M, Erlandsson R, Chiang S. Atomic-scale friction of a tungsten tip on a graphite surface. Phys Rev Lett 59(17): 1942-1945 (1987)

[14] Liu L, Zhou M, Jin L, Li L, Mo Y, Su G, Li X, Zhu H, Tian Y. Recent advances in friction and lubrication of graphene and other 2D materials: Mechanisms and applications. Friction 7(3): 199-216 (2019)

[15] Liu S-W, Wang H-P, Xu Q, Ma T-B, Yu G, Zhang C, Geng D, Yu Z, Zhang S, Wang W, et al. Robust microscale superlubricity under high contact pressure enabled by graphene-coated microsphere. Nat Commun 8(1): 14029 (2017)

[16] Wang W, Xie G, Luo J. Black phosphorus as a new lubricant. Friction 6(1): 116-142 (2018)

[17] Giessibl F J, Herz M, Mannhart J. Friction traced to the single atom. Proc Natl Acad Sci USA 99(19): 1200612010 (2002)

[18] Weymouth A J, Meuer D, Mutombo P, Wutscher T, Ondracek M, Jelinek P, Giessibl F J. Atomic structure affects the directional dependence of friction. Phys Rev Lett 111(12): 126103 (2013)

[19] Kawai S, Sasaki N, Kawakatsu H. Direct mapping of the lateral force gradient on $\mathrm{Si}(111)-7 \mathrm{x} 7$. Phys Rev B 79(19): 195412 (2009)

[20] Naitoh Y, Turansky R, Brndiar J, Li Y J, Stich I, Sugawara Y. Subatomic-scale force vector mapping above a $\mathrm{Ge}(001)$ dimer using bimodal atomic force microscopy. Nat Phys 13(7): 663-667 (2017)

[21] Umeda N, Ishizaki S, Uwai H. Scanning attractive force microscope using photothermal vibration. J Vac Sci Technol B 9(2): 1318-1322 (1991)

[22] Nishida S, Kobayashi D, Sakurada T, Nakazawa T, Hoshi Y, Kawakatsu H. Photothermal excitation and laser Doppler velocimetry of higher cantilever vibration modes for dynamic atomic force microscopy in liquid. Rev Sci Instrum 79(12): 123703 (2008)

[23] Tan X, Shi S, Guo D, Luo J. Dynamical characterization of micro cantilevers by different excitation methods in dynamic atomic force microscopy. Rev Sci Instrum 89(11): 115109 (2018)

[24] Rodriguez T R, Garcia R. Compositional mapping of surfaces in atomic force microscopy by excitation of the second normal mode of the microcantilever. Appl Phys Lett 84(3): 449-451 (2004)

[25] Lai C-Y, Santos S, Chiesa M. Systematic multidimensional quantification of nanoscale systems from bimodal atomic force microscopy data. Acs Nano 10(6): 6265-6272 (2016)

[26] Shi S, Guo D, Luo J. Enhanced phase and amplitude image contrasts of polymers in bimodal atomic force microscopy. RSC Adv 7(19): 11768-11776 (2017)

[27] Forchheimer D, Forchheimer R, Haviland D B. Improving image contrast and material discrimination with nonlinear response in bimodal atomic force microscopy. Nat Commun 6(1): 6270 (2015)

[28] Shi S, Guo D, Luo J. Interfacial interaction and enhanced image contrasts in higher mode and bimodal mode atomic force microscopy. RSC $A d v$ 7(87): 5512155130 (2017)

[29] Garcia R, Herruzo E T. The emergence of multifrequency force microscopy. Nat Nanotechnol 7(4): 217-26 (2012)

[30] Nievergelt A P, Adams J D, Odermatt P D, Fantner G E. High-frequency multimodal atomic force microscopy. Beilstein J Nanotechnol 5: 2459-2467 (2014)

[31] Platz D, Tholen E A, Pesen D, Haviland D B. Intermodulation atomic force microscopy. Appl Phys Lett 92(15): 153106 (2008)

[32] Lai C-Y, Perri S, Santos S, Garcia R, Chiesa M. Rapid quantitative chemical mapping of surfaces with sub-2 $\mathrm{nm}$ resolution. Nanoscale 8(18): 9688-9694 (2016) 
[33] Athanasopoulou E-N, Nianias N, Ong Q K, Stellacci F. Bimodal atomic force microscopy for the characterization of thiolated self-assembled monolayers. Nanoscale 10(48): 23027-23036 (2018)

[34] Benaglia S, Amo C A, Garcia R. Fast, quantitative and high resolution mapping of viscoelastic properties with bimodal AFM. Nanoscale 11(32): 15289-15297 (2019)

[35] Shi S, Guo D, Luo J B. Imaging contrast and tip-sample interaction of non-contact amplitude modulation atomic force microscopy with Q-control. J Phys D-Appl Phys 50(41): 415307 (2017)

[36] Kunstmann T, Schlarb A, Fendrich M, Paulkowski D, Wagner T, Moller R. Combined normal and torsional mode in frequency-modulation atomic force microscopy for lateral dissipation measurement. Appl Phys Lett 88(15): 153112 (2006)

[37] Yurtsever A, Gigler A M, Dietz C, Stark R W. Frequency modulated torsional resonance mode atomic force microscopy on polymers. Appl Phys Lett 92(14): 143103 (2008)

[38] Kawai S, Glatzel T, Koch S, Such B, Baratoff A, Meyer E. Ultrasensitive detection of lateral atomic-scale interactions on graphite $(0001)$ via bimodal dynamic force measurements. Phys Rev B 81(8): 085420 (2010)

[39] Dietz C. Sensing in-plane nanomechanical surface and sub-surface properties of polymers: local shear stress as function of the indentation depth. Nanoscale 10(1): 460-468 (2018)

[40] Tan X, Guo D, Luo J. Different directional energy dissipation of heterogeneous polymers in bimodal atomic force microscopy. $R S C A d v$ 9(47): 27464-27474 (2019)

[41] Socoliuc A, Gnecco E, Maier S, Pfeiffer O, Baratoff A, Bennewitz R, Meyer E. Atomic-scale control of friction by actuation of nanometer-sized contacts. Science 313(5784): 207-210 (2006)

[42] Lantz M A, Wiesmann D, Gotsmann B. Dynamic superlubricity and the elimination of wear on the nanoscale. Nat Nanotechnol 4(9): 586-591 (2009)

[43] Shi S, Guo D, Luo J. Micro/atomic-scale vibration induced superlubricity. Friction 9(5): 1163-1174 (2021)

[44] Forchheimer D, Forchheimer R, Haviland D B. Improving image contrast and material discrimination with nonlinear response in bimodal atomic force microscopy. Nat Commun 6(1): 6270 (2015)

[45] Ding R-F, Yang C-W, Huang K-Y, Hwang I-S. High-sensitivity imaging with lateral resonance mode atomic force microscopy. Nanoscale 8(43): 1842118427 (2016)

[46] Egberts P, Ye Z J, Liu X Z, Dong Y L, Martini A, Carpick R W. Environmental dependence of atomicscale friction at graphite surface steps. Phys Rev B 88(3): 035409 (2013)
[47] Martinez N F, Garcia R. Measuring phase shifts and energy dissipation with amplitude modulation atomic force microscopy. Nanotechnology 17(7): S167-S172 (2006)

[48] Sader J E, Chon J W M, Mulvaney P. Calibration of rectangular atomic force microscope cantilevers. Rev Sci Instrum 70(10): 3967-3969 (1999)

[49] Lozano J R, Garcia R. Theory of multifrequency atomic force microscopy. Phys Rev Lett 100(7): 076102 (2008)

[50] Santos S. Phase contrast and operation regimes in multifrequency atomic force microscopy. Appl Phys Lett 104(14): 143109 (2014)

[51] Garcia R, Gomez C J, Martinez N F, Patil S, Dietz C, Magerle R. Identification of nanoscale dissipation processes by dynamic atomic force microscopy. Phys Rev Lett 97(1): 016103 (2006)

[52] Garcia R, Tamayo J, San Paulo A. Phase contrast and surface energy hysteresis in tapping mode scanning force microsopy. Surf Interface Anal 27(5-6): 312-316 (1999)

[53] Chawla G, Solares S D. Mapping of conservative and dissipative interactions in bimodal atomic force microscopy using open-loop and phase-locked-loop control of the higher eigenmode. Appl Phys Lett 99(7): 074103 (2011)

[54] Lozano J R, Garcia R. Theory of phase spectroscopy in bimodal atomic force microscopy. Phys Rev B 79(1): 014110 (2009)

[55] Cleveland J P, Anczykowski B, Schmid A E, Elings V B. Energy dissipation in tapping-mode atomic force microscopy. Appl Phys Lett 72(20): 2613-2615 (1998)

[56] Gomez C J, Garcia R. Determination and simulation of nanoscale energy dissipation processes in amplitude modulation AFM. Ultramicroscopy 110(6): 626-633 (2010)

[57] Chen N, Bhushan B. Morphological, nanomechanical and cellular structural characterization of human hair and conditioner distribution using torsional resonance mode with an atomic force microscope. $J$ Microsc 220(2): 96-112 (2005)

[58] San Paulo A, Garcia R. Amplitude, deformation and phase shift in amplitude modulation atomic force microscopy: a numerical study for compliant materials. Surf Sci 471(1-3): 71-79 (2001)

[59] Israelachvili J N, Intermolecular and Surface Forces, 3rd Edition. San Diego: Elsevier Academic Press Inc, 2011.

[60] Ashman K M, Bird C M, Zepf S E. Detecting bimodality in astronomical datasets. Astron $J$ 108(6): 2348-2361 (1994)

[61] Stifter T, Marti O, Bhushan B. Theoretical investigation 
of the distance dependence of capillary and van der Waals forces in scanning force microscopy. Phys Rev $B$ 62(20): 13667-13673 (2000)

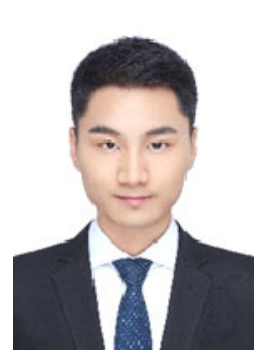

Xinfeng TAN. He obtained his B.E. degree from Beihang University in 2015, and received his Ph.D. degree in mechanical engineering in 2020 from Tsinghua University, China. He is working at State Key

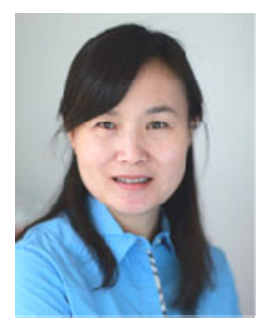

Dan GUO. She received the M.S. degree in engineering mechanics in 1995 from Xi'an Jiaotong University, Xi'an, China and Ph.D. degree in engineering mechanics in 1999 from Tsinghua University, Beijing, China. She joined the State Key Laboratory of Tribology at Tsinghua

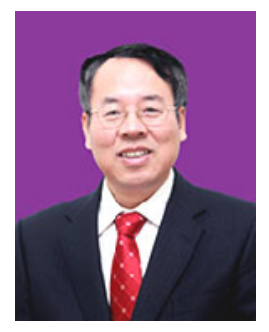

Jianbin LUO. He received his B.E. degree from Northeastern University in 1982, and got his M.E. degree from Xi'an University of Architecture and Technology in 1988. In 1994, he received his Ph.D. degree from Tsinghua University and then joined the faculty of Tsinghua University. Prof. Jianbin Luo is an academician of the Chinese Academy of Sciences and a Yangtze River Scholar
[62] Burnham N A, Colton R J, Pollock H M. Interpretation issues in force microscopy. J Vac Sci Technol A-Vac Surf Films 9(4): 2548-2556 (1991)

Laboratory of Tribology, Tsinghua University for postdoctoral research. His research interests include the dynamic friction energy dissipation detection at micro-nano scale as well as the design and improvement of micro-nano tribometer based on atomic force microscope.

University from 1999. Her current position is a professor and the deputy director of the laboratory. Her research areas cover the properties of friction at the micro/nano scale, mechanism of interaction among nanoparticles and surface in ultra-smooth surface planarization, and the formation and failure of lubricant film in harsh conditions.

Distinguished Professor of Tsinghua University, Beijing, China. He was awarded the STLE International Award (2013), the Chinese National Technology Progress Prize (2008), the Chinese National Natural Science Prize (2001), and the Chinese National Invention Prize (1996). Prof. Luo has been engaged in the research of thin film lubrication and tribology in nanomanufacturing. He was invited as a keynote or plenary speaker for 20 times on the international conferences. 